László Tóth

\title{
Another generalization of the ged-sum function
}

Received: 12 October 2012 / Accepted: 22 May 2013 / Published online: 5 June 2013

(C) The Author(s) 2013. This article is published with open access at Springerlink.com

Abstract We investigate an arithmetic function representing a generalization of the gcd-sum function, considered by Kurokawa and Ochiai in 2009 in connection with the multivariable global Igusa zeta function for a finite cyclic group. We show that the asymptotic properties of this function are closely connected to the Piltz divisor function. A generalization of Menon's identity is also considered.

Mathematics Subject Classification 11A25 - 11N37 · 11M32

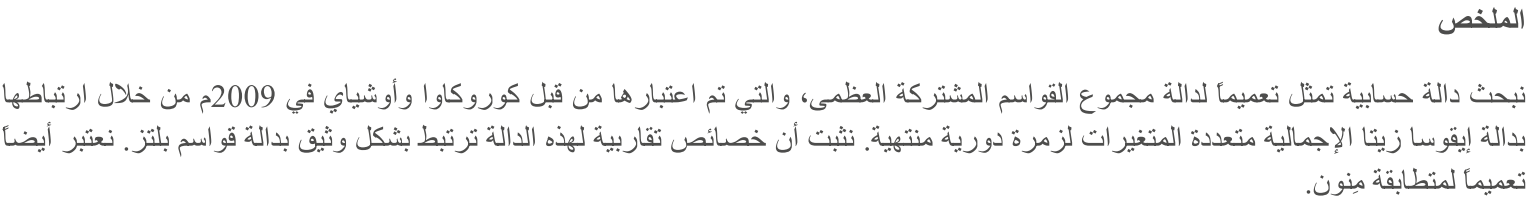

\section{Introduction}

Let $r \in \mathbb{N}:=\{1,2, \ldots\}$ and define the arithmetic function $A_{r}$ by

$$
A_{r}(n):=\frac{1}{n^{r}} \sum_{k_{1}, \ldots, k_{r}=1}^{n} \operatorname{gcd}\left(k_{1} \ldots k_{r}, n\right) \quad(n \in \mathbb{N}) .
$$

The function $A_{r}$ was considered by Kurokawa and Ochiai [6] in connection with certain zeta functions. More exactly, the multivariable global Igusa zeta function for a group $A$ is defined by

$$
Z^{\text {group }}\left(s_{1}, \ldots, s_{r} ; A\right):=\sum_{m_{1}, \ldots, m_{r}=1}^{\infty} \frac{\# \operatorname{Hom}\left(A, \mathbb{Z} / m_{1} \ldots m_{r} \mathbb{Z}\right)}{m_{1}^{s_{1}} \ldots m_{r}^{s_{r}}}
$$

L. Tóth $(\varangle)$

Department of Integrative Biology, Institute of Mathematics, Universität für Bodenkultur, Gregor Mendel-Straße 33,

1180 Vienna, Austria

E-mail: ltoth@gamma.ttk.pte.hu

L. Tóth

Department of Mathematics, University of Pécs, Ifjúság u. 6, Pécs 7624, Hungary 
Consider the case $A=\mathbb{Z} / n \mathbb{Z}(n \in \mathbb{N})$. Since the number of group homomorphisms $\mathbb{Z} / n \mathbb{Z} \rightarrow$ $\mathbb{Z} / m_{1} \ldots m_{r} \mathbb{Z}$ is $\operatorname{gcd}\left(n, m_{1} \ldots m_{r}\right)$, the function (1) reduces to

$$
Z^{\text {group }}\left(s_{1}, \ldots, s_{r} ; \mathbb{Z} / n \mathbb{Z}\right):=\sum_{m_{1}, \ldots, m_{r}=1}^{\infty} \frac{\operatorname{gcd}\left(m_{1} \ldots m_{r}, n\right)}{m_{1}^{s_{1}} \ldots m_{r}^{s_{r}}} .
$$

Kurokawa and Ochiai [6] derived two representations for (2), one of them being

$$
Z^{\text {group }}\left(s_{1}, \ldots, s_{r} ; \mathbb{Z} / n \mathbb{Z}\right)=\frac{1}{n^{s_{1}+\cdots+s_{r}}} \sum_{k_{1}, \ldots, k_{r}=1}^{\infty} \operatorname{gcd}\left(k_{1} \ldots k_{r}, n\right) \zeta\left(s_{1}, k_{1} / n\right) \ldots \zeta\left(s_{r}, k_{r} / n\right),
$$

where $\zeta(s, a):=\sum_{m=0}^{\infty} 1 /(m+a)^{s}$ denotes the Hurwitz zeta function. It follows from (3) that (2) has a meromorphic continuation to $\mathbb{C}^{r}$.

Proposition 1.1 $\left[6\right.$, Cor. 1] For every $n=\prod_{p \mid n} p^{v_{p}(n)} \in \mathbb{N}$,

$$
A_{r}(n)=\prod_{p \mid n} \sum_{j=0}^{r}\left(\left(\begin{array}{c}
v_{p}(n) \\
j
\end{array}\right)\right)\left(1-\frac{1}{p}\right)^{j}
$$

where

$$
\left(\left(\begin{array}{l}
n \\
k
\end{array}\right)\right):=\left(\begin{array}{c}
n+k-1 \\
k
\end{array}\right)=(-1)^{k}\left(\begin{array}{c}
-n \\
k
\end{array}\right)
$$

denotes the number of $k$-multisets of an $n$-set.

Proposition 1.2 [6, Cor. 2] For every $n \in \mathbb{N}$,

$$
\lim _{r \rightarrow \infty} A_{r}(n)=n .
$$

Formula (4) was obtained in [6] as an application of the representations given for (2), while (5) is a direct consequence of (4). Note that (4) was reproved in $[7,8]$ using the arguments of the elementary probability theory.

In the case $r=1$,

$$
A_{1}(n):=\frac{1}{n} \sum_{k=1}^{n} \operatorname{gcd}(k, n)=\sum_{d \mid n} \frac{\phi(d)}{d}
$$

where $\phi$ is Euler's totient function. Here $A_{1}(n)$ represents the arithmetic mean of $\operatorname{gcd}(1, n), \ldots, \operatorname{gcd}(n, n)$ and (4) reduces to

$$
A_{1}(n)=\prod_{p \mid n}\left(1+v_{p}(n)\left(1-\frac{1}{p}\right)\right) .
$$

See $[2,4,14,16]$ for various properties, analogs and other generalizations of the function (6).

In the present paper we derive a simple recursion formula for the functions $A_{r}$, offer a direct numbertheoretic proof for the formula (4) and show that the asymptotic properties of the function $A_{r}(n)$ are closely connected to the Piltz divisor function $\tau_{r+1}(n)$, defined as the number of ways of expressing $n$ as a product of $r+1$ factors.

As a modification of $A_{r}(n)$ we also consider and evaluate the function

$$
B_{r}(n):=\sum_{\substack{k_{1}, \ldots, k_{r}=1 \\ \operatorname{gcd}\left(k_{1} \ldots k_{r}, n\right)=1}}^{n} \operatorname{gcd}\left(k_{1} \ldots k_{r}-1, n\right) \quad(n, r \in \mathbb{N}) .
$$


Note that in the case $r=1$,

$$
B_{1}(n):=\sum_{\substack{k=1 \\ \operatorname{gcd}(k, n)=1}}^{n} \operatorname{gcd}(k-1, n)=\phi(n) \tau(n) \quad(n \in \mathbb{N}),
$$

where $\tau(n)$ stands for the number of divisors of $n$, according to a result of Menon [9]. See [12,15] for other Menon-type identities.

Our results are given in Sect. 2, while their proofs are included in Sect. 3.

\section{Results}

Let $A_{0}(n):=\mathbf{1}(n)=1(n \in \mathbb{N})$.

Proposition 2.1 The following recursion formula holds:

$$
A_{r}(n)=\sum_{d \mid n} \frac{\phi(d) A_{r-1}(d)}{d} \quad(n, r \in \mathbb{N}) .
$$

Let $\bar{\phi}(n)=\phi(n) / n$.

Corollary 2.2 In terms of the Dirichlet convolution, $A_{r}=\bar{\phi} A_{r-1} * \mathbf{1}(r \in \mathbb{N})$. Therefore, $A_{1}=\bar{\phi} * \mathbf{1}, A_{2}=$ $\bar{\phi}(\bar{\phi} * \mathbf{1}) * \mathbf{1}, A_{3}=\bar{\phi}(\bar{\phi}(\bar{\phi} * \mathbf{1}) * \mathbf{1}) * \mathbf{1}$, in general

$$
A_{r}=\bar{\phi}(\bar{\phi}(\ldots(\bar{\phi} * \mathbf{1}) \ldots) * \mathbf{1}) * \mathbf{1}
$$

including $r$ times $\bar{\phi}$ and $r$ times $\mathbf{1}$.

Corollary 2.3 The function $A_{r}$ is multiplicative for any $r \in \mathbb{N}$.

Observe that from formula (4),

$$
A_{r}(n) \leq \prod_{p \mid n} \sum_{j=0}^{r}\left(\begin{array}{c}
v_{p}(n)+j-1 \\
j
\end{array}\right)=\prod_{p \mid n}\left(\begin{array}{c}
v_{p}(n)+r \\
r
\end{array}\right)=\tau_{r+1}(n)
$$

for any $n \in \mathbb{N}$, using parallel summation of the binomial coefficients.

Also, $A_{r}\left(p^{k}\right)=\left(\begin{array}{c}k+r \\ r\end{array}\right)+\mathcal{O}(1 / p)=\tau_{r+1}\left(p^{k}\right)+\mathcal{O}(1 / p)$, as $p \rightarrow \infty$ ( $p$ prime) for any fixed $k, r \in \mathbb{N}$. This suggests that the asymptotic behavior of $A_{r}(n)$ is similar to that of $\tau_{r+1}(n)$.

Proposition 2.4 The Dirichlet series of the function $A_{r}$ has the representation

$$
\sum_{n=1}^{\infty} \frac{A_{r}(n)}{n^{s}}=\zeta^{r+1}(s) F_{r}(s) \quad(\Re(s)>1),
$$

where the Dirichlet series $F_{r}(s):=\sum_{n=1}^{\infty} f_{r}(n) / n^{s}$ is absolutely convergent for $\Re(s)>0$. Moreover, for any prime power $p^{k}, f_{r}\left(p^{k}\right)=0$ if $k \geq r+1$ and $f_{r}\left(p^{k}\right) \ll 1 / p$, as $p \rightarrow \infty$ if $1 \leq k \leq r$.

For the function $\tau_{k}(k \geq 2)$ one has

$$
\sum_{n \leq x} \tau_{k}(n)=\operatorname{Res}_{s=1} x^{s} \frac{\zeta^{k}(s)}{s}+\Delta_{k}(x)
$$

where the main term is $x P_{k-1}(\log x)$ with a suitable polynomial $P_{k-1}(t)$ in $t$ of degree $k-1$ having the leading coefficient $1 /(k-1)$ !. For the error term, $\Delta_{k}(x)=\mathcal{O}\left(x^{\alpha_{k}+\varepsilon}\right)$, with $\alpha_{k} \leq(k-1) /(k+1)(k \geq 2)$, $\alpha_{k} \leq(k-1) /(k+2)(k \geq 4)$. See [13, Ch. XII] and [5] for further results on $\Delta_{k}(x)$. 
Proposition 2.5 Let $r \in \mathbb{N}$. Then

$$
\sum_{n \leq x} A_{r}(n)=x Q_{r}(\log x)+R_{r}(x),
$$

where $Q_{r}(t)$ is a polynomial in $t$ of degree $r$ having the leading coefficient

$$
\frac{1}{r !} \prod_{p}\left(1+\sum_{k=1}^{r} \frac{f_{r}\left(p^{k}\right)}{p^{k}}\right)
$$

and $R_{r}(x)=\mathcal{O}\left(x^{\alpha_{r+1}+\varepsilon}\right)$ (valid for every $\left.\varepsilon>0\right)$.

Also, $R_{r}(x)=O\left(x^{r /(r+2)+\varepsilon}\right)$ and $R_{r}(x)=\Omega\left(b_{r}(x)\right)$, where

$$
b_{r}(x)=(x \log x)^{\frac{r}{2 r+2}}\left(\log _{2} x\right)^{\frac{r+2}{2 r+2}\left((r+1)^{(2 r+2) /(r+2)}-1\right)}\left(\log _{3} x\right)^{-\frac{3 r+2}{4 r+4}},
$$

$\log _{j}$ denoting the $j$-fold iterated logarithm.

Proposition 2.6 For every $r \in \mathbb{N}$,

$$
\limsup _{n \rightarrow \infty} \frac{\log A_{r}(n) \log \log n}{\log n}=\log (r+1) .
$$

In the case $r=1$, formulae (12), without the omega result, and (13) were obtained by Chidambaraswamy and Sitaramachandrarao [3, Th. 3.1, 4.1]. In fact, both results were proved in [3] for a slightly more general function, namely for $\psi_{k}(n)=\sum_{d \mid n} \phi_{k}(d) / d^{k}$, where $k \in \mathbb{N}$ and $\phi_{k}(n)=n^{k} \prod_{p \mid n}\left(1-1 / p^{k}\right)$ is the Jordan function of order $k$. Here $A_{1}(n)=\psi_{1}(n) / n$.

For the function $B_{r}(n)$ defined by (7) we have

Proposition 2.7 For every $n, r \in \mathbb{N}$,

$$
B_{r}(n)=\phi^{r}(n) \tau(n) .
$$

\section{Proofs}

Proof of Proposition 2.1

$$
A_{r}(n)=\frac{1}{n^{r}} \sum_{k_{1}, \ldots, k_{r}=1}^{n} \sum_{d \mid \operatorname{gcd}\left(k_{1} \ldots k_{r}, n\right)} \phi(d)=\frac{1}{n^{r}} \sum_{d \mid n} \phi(d) \sum_{\substack{k_{1}, \ldots, k_{r}=1 \\ k_{1} \ldots k_{r}=0(\bmod d)}}^{n} 1,
$$

where for fixed $k_{1}, \ldots, k_{r-1}$ the congruence $k_{1} \ldots k_{r-1} k_{r} \equiv 0(\bmod d)$ has $\operatorname{gcd}\left(k_{1} \ldots k_{r-1}, d\right)$ solutions $k_{r}$ $(\bmod d)$ and has $(n / d) \operatorname{gcd}\left(k_{1} \ldots k_{r-1}, d\right)$ solutions $k_{r}(\bmod d)$. Therefore,

$$
A_{r}(n)=\frac{1}{n^{r-1}} \sum_{d \mid n} \frac{\phi(d)}{d} \sum_{k_{1}, \ldots, k_{r-1}=1}^{n} \operatorname{gcd}\left(k_{1} \ldots k_{r-1}, d\right),
$$

and writing $k_{j}=d q_{j}+s_{j}$ with $1 \leq s_{j} \leq d, 0 \leq q_{j} \leq n / d-1(1 \leq j \leq r-1)$ we see that the inner sum is

$$
\sum_{\substack{1 \leq s_{1}, \ldots, s_{r-1} \leq d \\ 0 \leq q_{1}, \ldots, q_{r-1} \leq n / d-1}} \operatorname{gcd}\left(s_{1} \ldots s_{r-1}, d\right)=\left(\frac{n}{d}\right)^{r-1} d^{r-1} A_{r-1}(d),
$$

and inserting this into (14) we obtain (9). 
Proof of Proposition 1.1 The function $n \mapsto A_{r}(n)$ is multiplicative by Corollary 2.3. Therefore, to obtain (4) it is sufficient to consider the case $n=p^{k}(k \in \mathbb{N})$, a prime power. Let $x_{r}(k):=A_{r}\left(p^{k}\right)(r \geq 0)$ with a fixed prime $p$. From the recursion formula (9) we have

$$
A_{r}\left(p^{k}\right)=1+\sum_{j=1}^{k}\left(1-\frac{1}{p}\right) A_{r-1}\left(p^{j}\right)
$$

that is, by denoting $t:=1-1 / p$,

$$
x_{r}(k)=1+t \sum_{j=1}^{k} x_{r-1}(j) \quad(r, k \in \mathbb{N})
$$

where $x_{0}(k):=A_{0}\left(p^{k}\right)=1(k \in \mathbb{N})$. Here $x_{1}(k)=1+t \sum_{j=1}^{k} x_{0}(j)=1+k t, x_{2}(k)=1+t \sum_{j=1}^{k} x_{1}(j)=$ $1+t \sum_{j=1}^{k}(1+j t)=1+k t+\frac{k(k+1)}{2} t^{2}, x_{3}(k)=1+t \sum_{j=1}^{k} x_{2}(j)=1+t \sum_{j=1}^{k}\left(1+j t+\frac{j(j+1)}{2} t^{2}\right)=$ $1+k t+\frac{k(k+1)}{2} t^{2}+\frac{k(k+1)(k+2)}{6} t^{3}$.

We show by induction on $r$ that $x_{r}(k)$ is a polynomial in $t$ of degree $r$ with integer coefficients which do not depend on $r$, more exactly,

$$
x_{r}(k)=1+\sum_{i=1}^{r}\left(\left(\begin{array}{l}
k \\
i
\end{array}\right)\right) t^{i} .
$$

Assume that (16) is valid for $r$. Then by (15) we obtain for $r+1$,

$$
\begin{aligned}
x_{r+1}(k) & =1+t \sum_{j=1}^{k} x_{r}(j)=1+t \sum_{j=1}^{k}\left(1+\sum_{i=1}^{r}\left(\left(\begin{array}{l}
j \\
i
\end{array}\right)\right) t^{i}\right) \\
& =1+k t+\sum_{i=1}^{r} t^{i+1} \sum_{j=1}^{k}\left(\begin{array}{c}
j+i-1 \\
i
\end{array}\right)=1+\sum_{i=0}^{r}\left(\begin{array}{l}
k+i \\
i+1
\end{array}\right) t^{i+1} \\
& =1+\sum_{i=1}^{r+1}\left(\begin{array}{c}
k+i-1 \\
i
\end{array}\right) t^{i}=1+\sum_{i=1}^{r+1}\left(\left(\begin{array}{l}
k \\
i
\end{array}\right)\right) t^{i}
\end{aligned}
$$

applying the upper summation formula. This completes the proof of (4).

Proof of Proposition 2.4 We use the conventions $\left(\begin{array}{l}a \\ 0\end{array}\right)=1(a \in \mathbb{Z}),\left(\begin{array}{l}a \\ b\end{array}\right)=0(a, b \in \mathbb{N}, a<b)$. In terms of the Dirichlet convolution, $A_{r}=\tau_{r+1} * f_{r}, f_{r}=A_{r} * \mu^{(r+1)}$ with $\mu^{(r+1)}=\mu * \cdots * \mu(r+1$ times $)$, where $\mu^{(r+1)}\left(p^{k}\right)=(-1)^{k}\left(\begin{array}{c}r+1 \\ k\end{array}\right)$ for any prime power $p^{k}(k \in \mathbb{N})$.

Hence for any $k \in \mathbb{N}$,

$$
\begin{aligned}
f_{r}\left(p^{k}\right)=\sum_{\ell=0}^{k} \mu^{(r+1)}\left(p^{\ell}\right) A_{r}\left(p^{k-\ell}\right) & =\sum_{\ell=0}^{k}(-1)^{\ell}\left(\begin{array}{c}
r+1 \\
\ell
\end{array}\right) \sum_{j=0}^{r}\left(\begin{array}{c}
j+k-\ell-1 \\
j
\end{array}\right)\left(1-\frac{1}{p}\right)^{j} \\
& =\sum_{j=0}^{r}\left(1-\frac{1}{p}\right)^{j} \sum_{\ell=0}^{k}(-1)^{\ell}\left(\begin{array}{c}
r+1 \\
\ell
\end{array}\right)\left(\begin{array}{c}
j+k-\ell-1 \\
j
\end{array}\right),
\end{aligned}
$$

which is a polynomial in $1 / p$ of degree $r$.

Here for any $k \geq r+1$,

$$
f_{r}\left(p^{k}\right)=\sum_{j=0}^{r}\left(1-\frac{1}{p}\right)^{j} \sum_{\ell=0}^{r+1}(-1)^{\ell}\left(\begin{array}{c}
r+1 \\
\ell
\end{array}\right)\left(\begin{array}{c}
j+k-\ell-1 \\
j
\end{array}\right)=0,
$$


since $\left(\begin{array}{c}j+k-\ell-1 \\ j\end{array}\right)$ is a polynomial in $\ell$ of degree $j$ and the inner sum is zero for any $0 \leq j \leq r$ using the identity

$$
\sum_{\ell=0}^{n}(-1)^{\ell} \ell^{j}\left(\begin{array}{l}
n \\
\ell
\end{array}\right)=0 \quad(0 \leq j \leq n-1)
$$

Now for $1 \leq k \leq r$ we obtain from (17) that the constant term of the polynomial in $1 / p$ giving $f_{r}\left(p^{k}\right)$ is

$$
\begin{aligned}
c & :=\sum_{j=0}^{r} \sum_{\ell=0}^{k}(-1)^{\ell}\left(\begin{array}{c}
r+1 \\
\ell
\end{array}\right)\left(\begin{array}{c}
j+k-\ell-1 \\
j
\end{array}\right) \\
& =\sum_{\ell=0}^{k}(-1)^{\ell}\left(\begin{array}{c}
r+1 \\
\ell
\end{array}\right) \sum_{j=0}^{r}\left(\begin{array}{c}
j+k-\ell-1 \\
j
\end{array}\right) \\
& =\sum_{\ell=0}^{k}(-1)^{\ell}\left(\begin{array}{c}
r+1 \\
\ell
\end{array}\right)\left(\begin{array}{c}
r+k-\ell \\
r
\end{array}\right),
\end{aligned}
$$

using parallel summation again.

Using now that $\left(\begin{array}{c}r+k-\ell \\ r\end{array}\right)=(-1)^{k-\ell}\left(\begin{array}{c}-r-1 \\ k-\ell\end{array}\right)$ we obtain

$$
c=(-1)^{k} \sum_{\ell=0}^{k}\left(\begin{array}{c}
r+1 \\
\ell
\end{array}\right)\left(\begin{array}{c}
-(r+1) \\
k-\ell
\end{array}\right)=0
$$

by Vandermonde's identity.

Therefore, $f_{r}\left(p^{k}\right) \ll 1 / p$, as $p \rightarrow \infty$ for any $k \in\{1, \ldots, r\}$. This shows that the Dirichlet series $F_{r}(s)$ is absolutely convergent for $\Re(s)>0$.

Proof of Proposition 2.5 Using Proposition 2.4 and (11) for $k=r+1$,

$$
\begin{aligned}
\sum_{n \leq x} A_{r}(n) & =\sum_{d \leq x} f_{r}(d) \sum_{e \leq x / d} \tau_{r+1}(e) \\
& =\sum_{d \leq x} f_{r}(d)\left(\frac{x}{d} P_{r}(\log (x / d))+\Delta_{r+1}(x / d)\right),
\end{aligned}
$$

and (12) follows by usual estimates.

To obtain the omega result let $g_{r}$ denote the inverse under Dirichlet convolution of the function $f_{r}$. Then $g_{r}$ is multiplicative, $\tau_{r+1}=g_{r} * A_{r}$, so that

$$
\sum_{n \leq x} \tau_{r+1}(n)=\sum_{d \leq x} g_{r}(d) \sum_{e \leq x / d} A_{r}(e)
$$

and the Dirichlet series $\sum_{n=1}^{\infty} g_{r}(n) / n^{s}$ is absolutely convergent for $\Re(s)>0$. Now apply the $\Omega$-result concerning the function $\tau_{k}$, due to Soundararajan [10], for $k=r+1$. In the case $r=1$,

$$
\sum_{n \leq x} \tau(n)=\sum_{d \leq x} \frac{1}{d} \sum_{e \leq x / d} A_{1}(e)=x \log x+(2 \gamma-1) x+\sum_{d \leq x} \frac{1}{d} R_{1}(x / d)+O(\log x) .
$$

Assume that $R_{1}(x)=\Omega\left(b_{1}(x)\right)$ does not hold. Then for every $c>0$ there exists $x_{c}>0$ such that $\left|R_{1}(x)\right| \leq c b_{1}(x)$ for any $x \geq x_{c}$. Now inserting this into (18) contradicts that $\Delta(x)=\Omega(b(x))$. The same proof works out also for $r \geq 2$. 
Proof of Proposition 2.6 Similar to the proof of [3, Th. 4.1]. By (10), $A_{r}(n) \leq \tau_{r+1}(n)(n \in \mathbb{N})$. Therefore, using that (13) holds for $\tau_{r+1}(n)$ instead of $A_{r}(n)$ [11, Eq. 3.4] we obtain that the given lim sup is $\leq \log (r+1)$.

Furthermore, for squarefree $n$,

$$
\begin{aligned}
A_{r}(n)=\prod_{p \mid n} \sum_{j=0}^{r}(1-1 / p)^{j} & =\prod_{p \mid n} p\left(1-(1-1 / p)^{r+1}\right) \\
& =\prod_{p \mid n}\left(r+1-\frac{r(r+1)}{2} \cdot \frac{1}{p}+O\left(1 / p^{2}\right)\right) \\
& =(r+1)^{\omega(n)} \prod_{p \mid n}\left(1-\frac{r}{2} \cdot \frac{1}{p}+O\left(1 / p^{2}\right)\right),
\end{aligned}
$$

as $p \rightarrow \infty$ (for every fixed $r$ ).

Let $n_{x}=\prod_{x / \log x<p \leq x} p$. Then

$$
\begin{aligned}
& \frac{\log A_{r}\left(n_{x}\right) \log \log n_{x}}{\log n_{x}} \\
& =\log (r+1) \frac{\omega\left(n_{x}\right) \log \log n_{x}}{\log n_{x}}+\frac{\log \log n_{x}}{\log n_{x}} \log \prod_{p \mid n_{x}}\left(1-\frac{r}{2} \cdot \frac{1}{p}+O\left(1 / p^{2}\right)\right) .
\end{aligned}
$$

By using familiar estimates, $\log n_{x} \sim x, \log \log n_{x} \sim \log x$ and $\omega\left(n_{x}\right) \sim x / \log x$. Hence $\omega\left(n_{x}\right) \log \log n_{x} / \log n_{x} \rightarrow 1$, as $x \rightarrow \infty$.

Also, $\prod_{p \leq x}\left(1-\frac{r}{2} \cdot \frac{1}{p}+O\left(1 / p^{2}\right)\right) \sim C_{r} /(\log x)^{r / 2}$ with a suitable constant $C_{r}$. Therefore, $\prod_{p \mid n_{x}}\left(1-\frac{r}{2} \cdot \frac{1}{p}+O\left(1 / p^{2}\right)\right) \rightarrow 1$ as $x \rightarrow \infty$, and the result follows.

Proof of Proposition 2.7 We use the following lemma, which follows easily by the inclusion-exclusion principle (cf. [1, Th. 5.32]).

Lemma 3.1 Let $n, d, x \in \mathbb{N}$ be such that $d \mid n, 1 \leq x \leq d, \operatorname{gcd}(x, d)=1$. Then

$$
\#\{k \in \mathbb{N}: 1 \leq k \leq n, k \equiv x(\bmod d), \operatorname{gcd}(k, n)=1\}=\phi(n) / \phi(d) .
$$

We also need the following identity, which reduces to (8) in the case $a=1$.

Lemma 3.2 Let $\operatorname{gcd}(a, n)=1$. Then

$$
\sum_{\substack{k=1 \\ \operatorname{gcd}(k, n)=1}}^{n} \operatorname{gcd}(a k-1, n)=\phi(n) \tau(n) \quad(n \in \mathbb{N})
$$

For the proof of Lemma 3.2 write

$$
\sum_{\substack{k=1 \\ \operatorname{gcd}(k, n)=1}}^{n} \operatorname{gcd}(a k-1, n)=\sum_{\substack{k=1 \\ \operatorname{gcd}(k, n)=1}}^{n} \sum_{d \mid \operatorname{gcd}(a k-1, n)} \phi(d)=\sum_{d \mid n} \phi(d) \sum_{\substack{1 \leq k \leq n \\ \operatorname{gcd}(k, n)=1 \\ a k=1(\bmod d)}} 1
$$

and observe that for every $d \mid n$ the congruence $a k \equiv 1(\bmod d)$ has a unique solution $(\bmod d)$, since $\operatorname{gcd}(a, n)=1$. Therefore the inner sum is $\phi(n) / \phi(d)$ by Lemma 3.1. See also [15, Cor. 14].

Now for the proof of Proposition 2.7,

$$
B_{r}(n)=\sum_{\substack{k_{1}, \ldots, k_{r-1}=1 \\ \operatorname{gcd}\left(k_{1} \ldots k_{r-1}, n\right)=1}}^{n} \sum_{\substack{k_{r}=1 \\ \operatorname{gcd}\left(k_{r}, n\right)=1}}^{n} \operatorname{gcd}\left(\left(k_{1} \ldots k_{r-1}\right) k_{r}-1, n\right),
$$


and applying Lemma 3.2 for $a=k_{1} \ldots k_{r-1}$ we obtain that the inner sum is $\phi(n) \tau(n)$. Hence,

$$
B_{r}(n)=\sum_{\substack{k_{1}, \ldots, k_{r-1}=1 \\ \operatorname{gcd}\left(k_{1} \ldots k_{r-1}, n\right)=1}}^{n} \phi(n) \tau(n)=\phi^{r}(n) \tau(n) .
$$

Acknowledgments The author gratefully acknowledges support from the Austrian Science Fund (FWF) under the projects No. P20847-N18 and M1376-N18. The author thanks Professor Werner Georg Nowak for very helpful discussions on the subject.

Open Access This article is distributed under the terms of the Creative Commons Attribution License which permits any use, distribution, and reproduction in any medium, provided the original author(s) and the source are credited.

\section{References}

1. Apostol, T.M.: Introduction to Analytic Number Theory. Springer, Berlin (1976)

2. Chen, S.; Zhai, W.: Reciprocals of the gcd-sum functions. J. Integer. Seq. 14, Article 11.8.3 (2011)

3. Chidambaraswamy, J.; Sitaramachandrarao, R.: Asymptotic results for a class of arithmetical functions. Monatsh. Math. 99, 19-27 (1985)

4. de Koninck, J.-M.; Kátai, I.: Some remarks on a paper of L. Toth. J. Integer Seq. 13, Article 10.1.2 (2010)

5. Ivić, A.; Krätzel, E.; Kühleitner, M.; Nowak, W.G.: Lattice Points in Large Regions and Related Arithmetic Functions: Recent Developments in a Very Classic Topic. Elementare und Analytische Zahlentheorie. Schr. Wiss. Ges. Johann Wolfgang Goethe Univ. Frankfurt am Main., vol. 20, pp. 89-128. Franz Steiner Verlag Stuttgart, Stuttgart (2006)

6. Kurokawa, N.; Ochiai, H.: A multivariable Euler product of Igusa type and its applications. J. Number Theory 129, 1919-1930 (2009)

7. Minami, N.: On the random variable $\mathbb{N}^{r} \ni\left(k_{1}, k_{2}, \ldots, k_{r}\right) \mapsto \operatorname{gcd}\left(n, k_{1} k_{2} \ldots k_{r}\right) \in \mathbb{N}$. Preprint (2009). http://arxiv.org/ abs/0907.0916

8. Minami, N.: On the random variable $\{1,2, \ldots, n\}^{r} \ni\left(k_{1}, k_{2}, \ldots, k_{r}\right) \mapsto \operatorname{gcd}\left(n, k_{1} k_{2} \ldots k_{r}\right) \in \mathbb{N}$. J. Number Theory 133, 2635-2647 (2013)

9. Menon, K.P.: On the sum $\sum(a-1, n)[(a, n)=1]$. J. Indian Math. Soc. (N.S.). 29, 155-163 (1965)

10. Soundararajan, K.: Omega results for the divisor and circle problems. Int. Math. Res. Not. 36, 1987-1998 (2003)

11. Suryanarayana, D.; Sitaramachandrarao, R.: On the true maximum order of a class of arithmetical functions. Math. J. Okayama Univ. 17, 95-101 (1975)

12. Tărnăuceanu, M.: A generalization of Menon's identity. J. Number Theory 132, 2568-2573 (2012)

13. Titchmarsh, E.C.: The Theory of the Riemann Zeta-Function, 2nd edn. Oxford University Press, NY (1986) (revised by D.R. Heath-Brown)

14. Tóth, L.: A survey of gcd-sum functions. J. Integer Seq. 13, Article 10.8.1 (2010)

15. Tóth, L.: Menon's identity and arithmetical sums representing functions of several variables. Rend. Sem. Mat. Univ. Politec. Torino 69, 97-110 (2011)

16. Zhang, D.; Zhai, W.: Mean values of a class of arithmetical functions. J. Integer Seq. 14, Article 11.6 .5 (2011) 